\title{
High-Resolution Neurometabolic Coupling in the Lateral Geniculate Nucleus
}

\author{
Baowang Li and Ralph D. Freeman \\ Vision Science Group, School of Optometry, and Helen Wills Neuroscience Institute, University of California, Berkeley, Berkeley, California 94720-2020
}

\begin{abstract}
The relationships between neural and metabolic processes in activated brain regions are central to the interpretation of noninvasive imaging. To examine this relationship, we have used a specialized sensor to measure simultaneously tissue oxygen changes and neural activity in colocalized regions of the cat's lateral geniculate nucleus (LGN). Previous work with this sensor has shown that a decrease or increase in tissue oxygen can be elicited by selective control of the location and extent of neural activation in the LGN. In the current study, to evaluate the temporal integration and homogeneity of neurometabolic coupling, we have determined the relationship between multiunit extracellular neural activity and tissue oxygen responses to visual stimuli of various durations and contrasts. Our results show that the negative but not the positive oxygen response changes in an approximately linear manner with stimulus duration. The relationship between the negative oxygen response and neural activity is relatively constant with stimulus duration. Moreover, both negative and positive oxygen responses saturate at high stimulus contrast levels. Coupling between neural activity and negative oxygen responses is well described by a power law function. These results help elucidate differences between the initial negative and subsequent positive metabolic responses and may be directly relevant to questions concerning brain mapping with functional magnetic resonance imaging.
\end{abstract}

Key words: tissue oxygen; neural activity; linear and nonlinear; neurometabolic coupling; LGN; vision

\section{Introduction}

Functional neuroimaging techniques, such as functional magnetic resonance imaging (fMRI), infer neural activity from changes in hemodynamics. Most fMRI studies use the blood oxygen level-dependent (BOLD) signal and assume that cerebral blood flow $(\mathrm{CBF})$ underlies the localization of particular areas involved in a task. However, the use of spatial and temporal characteristics of the positive BOLD response to estimate neural activity is not straightforward (Grinvald et al., 2000; Logothetis et al., 2001). Studies in which fMRI or optical imaging techniques are used, show that the early transit increase in deoxyhemoglobin (Hbr; initial dip) is well localized to the presumed sites of neural activity (Malonek and Grinvald, 1996; Duong et al., 2000; Kim et al., 2000; Kim and Duong, 2002). Recent studies in which colocalized measurements were made of oxygen concentration and neural activity confirm on a fine spatial scale that the initial negative response is more spatially localized (Thompson et al., 2004, 2005) and that neural activity is coupled with the initial negative oxygen change rather than the subsequent positive oxygen response (Thompson et al., 2003, 2004, 2005). These results suggest that the initial negative component may be very useful in the application of high-resolution fMRI (Duong et al., 2000; Kim et al., 2000; Kim and Duong, 2002). However, the initial dip in fMRI is controversial. It is generally weak and variable and is often not

\footnotetext{
Received April 4, 2007; revised July 9, 2007; accepted Aug. 1, 2007. This work was supported by National Eye Institute Grants EY01175 and EY03176.

Correspondence should be addressed to Dr. Ralph D. Freeman, 360 Minor Hall, University of California, Berkeley, Berkeley, CA 94720-2020. E-mail: freeman@neurovision.berkeley.edu.

D0I:10.1523/JNEUROSCI.1505-07.2007

Copyright $\odot 2007$ Society for Neuroscience $\quad 0270-6474 / 07 / 2710223-07 \$ 15.00 / 0$
}

observed. But because it has the potential to increase the resolution of fMRI, it is worth exploring the initial dip in detail.

Conventional fMRI techniques are based on the positive BOLD signal and assume a linear relationship between neural activity and hemodynamic changes (Boynton et al., 1996; Logothetis et al., 2001; Heeger and Ress, 2002; Logothetis, 2002). However, recent optical imaging studies bring into question this assumption (Devor et al., 2003; Sheth et al., 2004). In contrast to the positive BOLD signal, the initial dip that is sometimes observed in $\mathrm{MRR}$ and optical imaging studies is thought to reflect localized neural activity (Malonek and Grinvald, 1996; Yacoub and $\mathrm{Hu}, 1999,2001$; Yacoub et al., 1999; Duong et al., 2000; Jones et al., 2001; Kim and Duong, 2002). However, basic characteristics of the initial dip remain unclear. To clarify the relationship between the initial dip of the fMRI signal and neural activity, metabolic and neural functions should be measured simultaneously. This may be accomplished indirectly by determinations of oxygen consumption during neural activation in a given region of the cerebral cortex.

Tissue oxygen measurements usually exhibit an early negative component followed by a positive response (Thompson et al., 2003; Offenhauser et al., 2005). In recent studies, by taking advantage of the precise retinotopic organization of the lateral geniculate nucleus (LGN) to control the extent and pattern of neural activity, monophasic negative and positive oxygen responses have been observed. In the current study, by using this technique, we have measured neural and oxygen responses at different stimulus durations and contrast levels to evaluate the extent of linearity of both oxygen response components. 


\section{Materials and Methods}

Physiological preparation. All procedures complied with the National Institutes of Health Guide for the Care and Use of Laboratory Animals. A total of 23 cats (5-8 months of age; $2.5-3.5 \mathrm{~kg}$ ) were used in this study. Anesthesia was induced with isoflurane, and femoral veins were cannulated. A thermistor probe was inserted to monitor core body temperature that was maintained at $38^{\circ} \mathrm{C}$. A tracheostomy was performed, and a tracheal cannula was positioned. Isoflurane was discontinued, and anesthesia was continued with thiopental sodium delivered intravenously combined with fentanyl $(10 \mu \mathrm{g} / \mathrm{h})$. After surgical procedures, fentanyl was discontinued and thiopental was set for continuous infusion as determined individually for each cat (generally $1.0-2.5 \mathrm{mg} \cdot \mathrm{kg}^{-1} \cdot \mathrm{h}^{-1}$ ). The animal was artificially ventilated $\left(25 \% \mathrm{O}_{2}-75 \% \mathrm{~N}_{2} \mathrm{O}\right)$ at a rate adjusted to maintain expired $\mathrm{CO}_{2}$ at $4-5 \%$. A craniotomy was performed at $6 \mathrm{~mm}$ anterior and $9 \mathrm{~mm}$ lateral from zero Horsley-Clarke coordinates. The dura was resected and covered with agar and wax to prevent drying of the cortex surface and to reduce pulsation. After the anesthetic level was stabilized at a constant rate of thiopental sodium, the cat was paralyzed with an intravenous infusion of pancuronium bromide $(0.2$ $\left.\mathrm{mg} \cdot \mathrm{kg}^{-1} \cdot \mathrm{h}^{-1}\right)$. Electroencephalogram, electrocardiogram, heart rate, temperature, intratracheal pressure, and end-tidal $\mathrm{CO}_{2}$ were monitored during the experiment.

Tissue oxygen signal and neural activity. A Clark-style polarographic oxygen sensor and an adjacent platinum microelectrode housed within a double-barrel micropipette were used to record neural activity and oxygen response (Unisense, Aarhus, Denmark). The tip diameter was $\sim 30$ $\mu \mathrm{m}$, and the field of sensitivity of the oxygen microsensor was a sphere of $\sim 60 \mu \mathrm{m}$ ( $2 \times$ tip diameter). Oxygen microsensors were calibrated in a bath of $0.9 \%$ saline at $38^{\circ} \mathrm{C}$ and exhibited linear oxygen response to changes in oxygen concentration. The oxygen sensor was connected to a high-impedance picoammeter (PA 2000; Unisense). Oxygen signals were sampled at $10 \mathrm{~Hz}$. In total, three different electrode units were used for the experiments reported here.

The microelectrode unit was advanced vertically into the LGN (Horsley-Clarke coordinates: $6 \mathrm{~mm}$ anterior and $9 \mathrm{~mm}$ lateral from zero) at a depth of $\sim 12 \mathrm{~mm}$ from the cortical surface by use of an electric micrometer. The impedance of the neural electrodes was between 0.66 and 1.0 $\mathrm{M} \Omega$ (mean, $0.84 \pm 0.17 \mathrm{M} \Omega ; n=3$ ). The preamplified signal $(10 \times)$ was amplified $(2000 \times)$ and filtered between 0.25 and $8 \mathrm{kHz}$. Extracellular action potentials were discriminated by setting a threshold above the background noise and producing multiunit neural activity (MUA). Drifting sinusoidal gratings were used as the visual stimulus. Custom-made search software was used to provide a display of a variety of visual stimulus parameters. Following preliminary estimates of optimal stimulus parameters, tuning functions were obtained quantitatively with drifting sinusoidal gratings of $50 \mathrm{~cd} / \mathrm{m}^{2}$ mean luminance. The visual stimuli were optimized for spatial and temporal frequency. The stimuli positioned on the receptive field (RF) were presented monoptically to the dominant eye while the unstimulated eye viewed a blank screen (mean luminance, $50 \mathrm{~cd} / \mathrm{m}^{2}$ ). The positions of RFs were remapped approximately every hour and adjusted if any change occurred.

Recording sites with minimal spontaneous oscillations in oxygen signal were chosen for extended study. By using this standard, $\sim 20 \%$ of potential recording sites were excluded because of large oscillations. Depending on the signal-to-noise ratio of the oxygen responses at each recording site, the influence of oscillations was reduced by averaging across multiple trials (from 16 to 64 ) of a given stimulus condition. The interstimulus interval was randomized between 30 and $44 \mathrm{~s}$ to avoid synchronization with oscillations. The spontaneous oxygen signal (assessed at $10 \mathrm{~s}$ before stimulus onset) was subtracted from individual oxygen responses. Oxygen responses were averaged and divided by the mean oxygen level across all trials and are shown here as percentages of oxygen change (Thompson et al., 2003, 2004, 2005).

\section{Results}

We used two separate protocols to investigate quantitatively details of neural and oxygen response relationships at recording sites in the LGN. These protocols take advantage of the clear
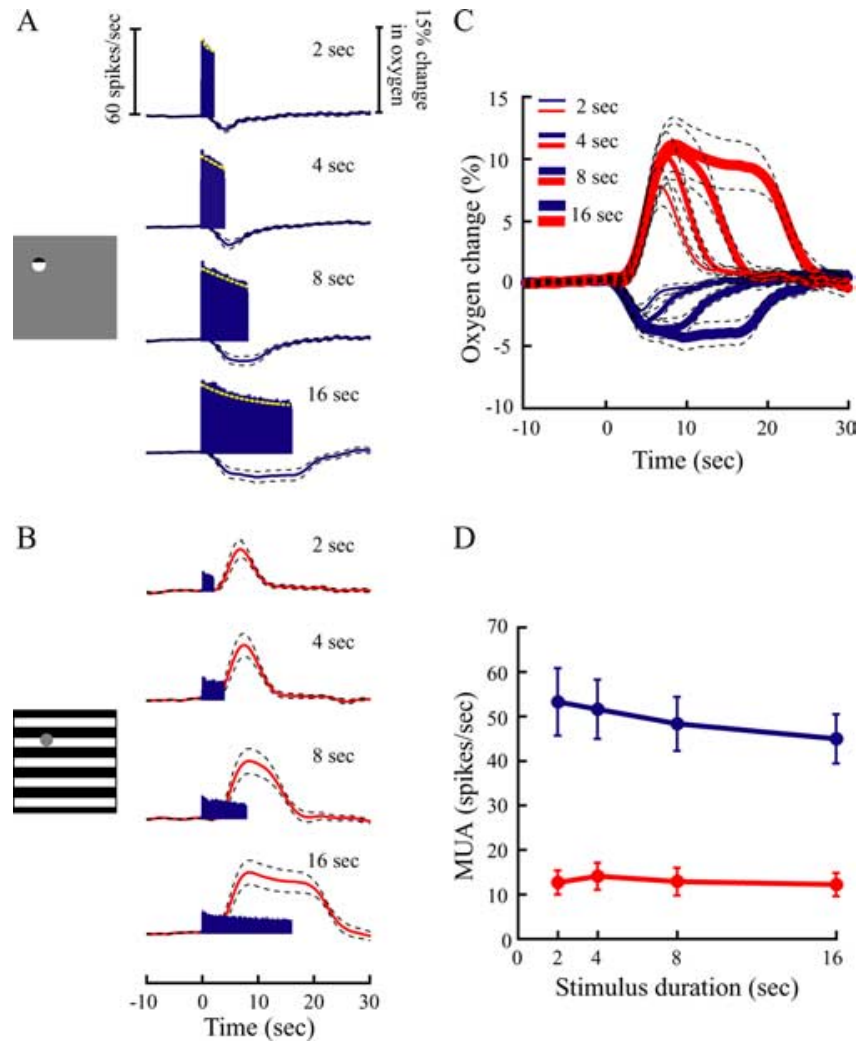

D

Figure 1. Average oxygen responses and neuronal activity to visual stimuli of different durations are illustrated for a population of recording sites $(n=27)$ in the LGN. $\boldsymbol{A}$, Neural and oxygen responses to the RF stimulus. The blue curves and histograms represent oxygen responses and neural activity. Vertical scale bars represent neural firing rates (left) and percentage change of oxygen signal (right). Dashed lines represent \pm 1 SE. Dashed yellow curves represent estimated neural responses by fitting corresponding histograms with an exponent function: $r=c+b \times \exp (-t / n)$, where $r$ is the neural response; $t$ is time; and $c, b$, and $n$ are free parameters (Soltysik et al., 2004). The goodness of fit is $0.68 \pm 0.18$ (SD) $(n=4)$. $\boldsymbol{B}$, Neural (histograms) and oxygen (red curves) responses to the large stimulus that extends outside the RF. $\boldsymbol{C}$, The negative (blue) and positive (red) curves represent oxygen responses to small and large stimuli, respectively, at different durations. Data are identical to those in $\boldsymbol{A}$ and $\boldsymbol{B}$. $\boldsymbol{D}$, Average neural responses to the small stimulus (blue dots) and the large stimulus (red dots) at different durations.

retinotopic neuronal organization of the LGN, the small RFs, and the relatively vigorous discharge rates of spikes. We recorded from 48 sites, of which 27 were used to investigate temporal integration properties. In these experiments, we sought to determine whether oxygen responses, both negative and positive, are linearly related in time. Specifically, are long-duration stimuli responses related linearly to those of a brief nature? In addition, we wanted to know whether neurometabolic coupling is time invariant with stimulus duration. For the remaining 21 recording sites, we investigated homogeneity. Specifically, we tested whether oxygen responses, both negative and positive, have a linear relationship with stimulus contrast. We also tested the notion that negative oxygen responses are linearly coupled with neural activity at different stimulus contrast levels. For both protocols, we only used responses that were stable over long periods. All recording sites we used (around half of those we encountered) were stable for $\geq 3 \mathrm{~h}$ such that response properties were relatively constant.

\section{Temporal integration}

We varied the duration of two stimulus patterns (Fig. $1 A, B$ ) to conduct the protocols noted above. In both cases, we recorded 

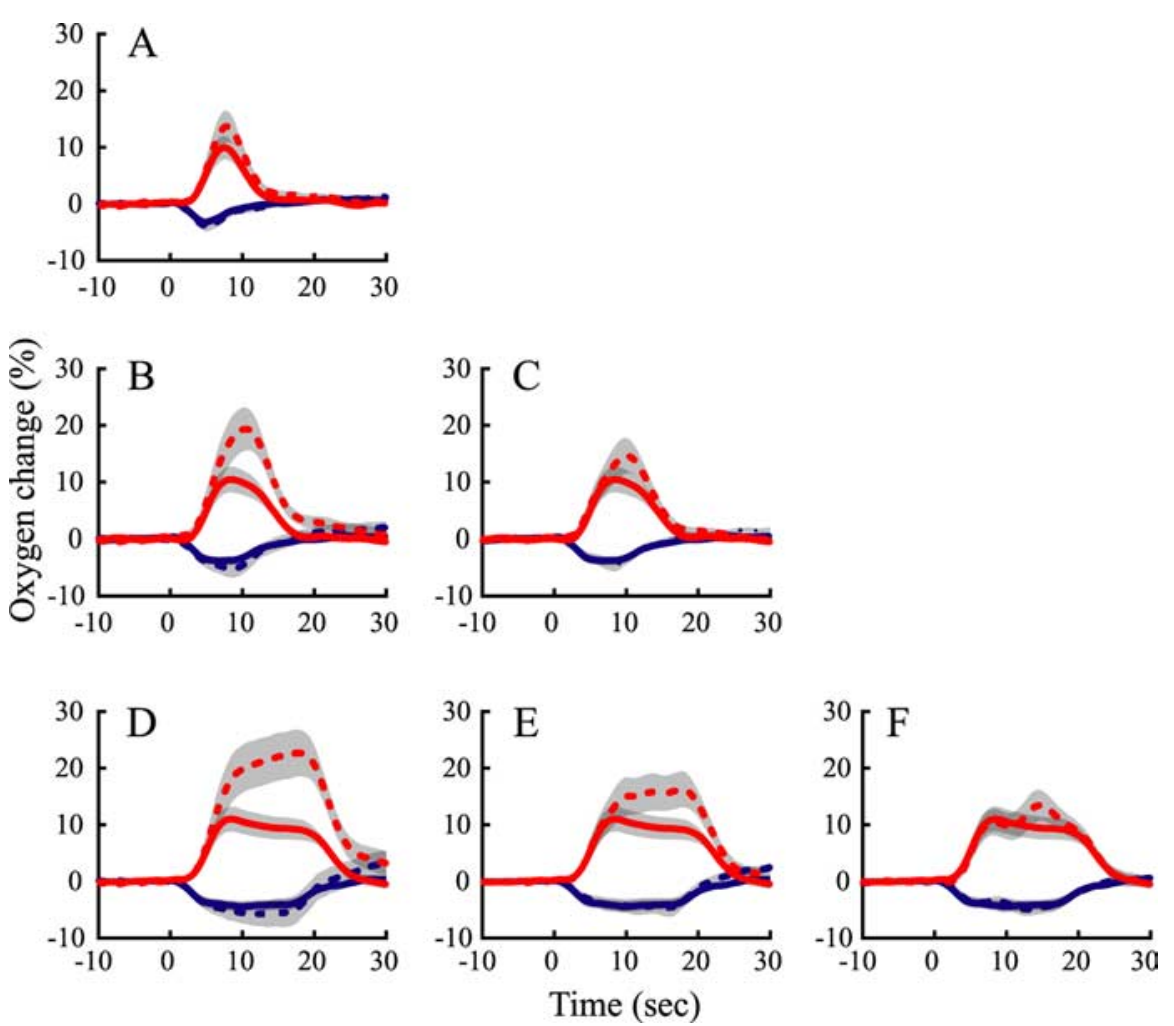

Figure 2. Time-invariant analysis of oxygen responses. Predicted responses to long-duration stimuli are generated by replicating, shifting, and summing measured responses to short-duration stimuli. Oxygen responses to four stimulus durations are used to make six predictions. $\boldsymbol{A}$, Measured (solid curves) and predicted (dashed curves) oxygen responses to $4 \mathrm{~s}$ stimuli based on responses to $2 \mathrm{~s}$ stimuli. Blue and red curves represent oxygen responses to the small and large visual stimuli, respectively. Gray filled areas represent \pm 1 SE. $\boldsymbol{B}, \boldsymbol{C}$, Measured and predicted oxygen responses to $8 \mathrm{~s}$ stimuli based on responses to $2 \mathrm{~s}(\boldsymbol{B})$ and $4 \mathrm{~s}$ (C) stimuli. $\boldsymbol{D}-\boldsymbol{F}$, Measured and predicted oxygen responses to $16 \mathrm{~s}$ stimuli based on responses to $2 \mathrm{~s}(\boldsymbol{D}), 4 \mathrm{~s}(\boldsymbol{E})$, and $8 \mathrm{~s}(\boldsymbol{F})$ stimuli.

stimulus-induced oxygen and multiunit neural responses in the LGN. Mean neural and oxygen responses from a population of recording sites $(n=27)$ are shown in Figure 1 . The small stimulus was a drifting grating ( $100 \%$ contrast) that was centered over the $\mathrm{RF}$ of the recording site (Fig. $1 A$ ). This stimulus is generally $1-3^{\circ}$, as determined by size tuning measurements to establish the strongest neural responses. For all presentation durations $(2,4,8$, and $16 \mathrm{~s})$, this stimulus elicited vigorous neural activity and monophasic negative oxygen responses as shown by the poststimulus time histogram spike patterns and oxygen response curves, respectively. The amplitude and width of the negative oxygen responses increase monotonically with stimulus duration. The large stimulus (Fig. $1 B$ ) was a full-field drifting grating (100\% contrast) with blank mask centered over the RF of the recording site. The intention here is the complement of the first stimulus (i.e., we are activating the region surrounding the RF). For all durations, this large surround stimulus elicited weak or no neural activity, which is expected because the stimulus was mainly outside the RF. Oxygen responses were either monophasic positive or weakly biphasic. The amplitude and width of the positive oxygen response increases monotonically with stimulus duration. Note that the large surround stimulus is outside the classical RF so that the weak spike discharge observed is likely to be dissociated from the oxygen responses (Legatt et al., 1980; Thompson et al., 2005).

To enable visual comparisons, negative and positive oxygen responses are plotted together in Figure $1 C$. It is evident that the amplitudes and widths of both the negative and positive oxygen signals increase monotonically with stimulus duration. Mean neural activity (MUA) is shown as a function of stimulus duration in Figure $1 D$. Clearly, MUA associated with the positive oxygen response remains essentially constant with stimulus duration (red line). In contrast, there is a slight gradual decline in MUA with duration for the negative oxygen response (blue line). This decrease is not statistically significant $(p=0.81$, ANOVA). To determine whether the increase in oxygen response is linearly related to stimulus duration, we made the following comparisons. Responses to long-duration stimuli are compared with those predicted by summing shifted replicas of the responses to short-duration stimuli to match the durations of longer stimuli. Stimulus durations of 2, 4, 8, and $16 \mathrm{~s}$ provide six predictions. Responses to the $4 \mathrm{~s}$ stimuli are predicted by summing responses to the $2 \mathrm{~s}$ stimuli with these right-shifted by $2 \mathrm{~s}$ (Fig. $2 \mathrm{~A}$, dashed lines). Likewise, responses to the $8 \mathrm{~s}$ stimuli are predicted by summing four shifted copies of the responses to the $2 \mathrm{~s}$ stimuli (Fig. $2 \mathrm{~B}$ ) or two shifted copies of the responses to the 4 s stimuli (Fig. 2C). Responses to the $16 \mathrm{~s}$ stimuli are predicted by summing eight shifted copies of the responses to the $2 \mathrm{~s}$ stimuli (Fig. 2D), four shifted copies of the responses to the $4 \mathrm{~s}$ stimuli (Fig. $2 E$ ), or two shifted copies of the responses to the $8 \mathrm{~s}$ stimuli (Fig. $2 F$ ). For the negative oxygen response, the predicted and measured oxygen responses are approximately consistent with a linear prediction. For the positive oxygen response, however, different amounts of overprediction are generally observed. Responses to long-duration stimuli (such as $8 \mathrm{~s}$ ) tend to linearly predict the responses to longer-duration stimuli (such as $16 \mathrm{~s}$ ) (Fig. $2 \mathrm{~F}$ ), whereas responses to short-duration stimuli (such as $2 \mathrm{~s}$ ) tend to overpredict responses to longer-duration stimuli (such as $16 \mathrm{~s}$ ) (Fig. 2D). The departure from linearity of the positive oxygen response to short-duration stimuli is consistent with a similar finding for the BOLD signal in fMRI studies (Boynton et al., 1996; Liu and Gao, 2000; Miller et al., 2001).

An alternative way to quantify the linearity of oxygen response is to examine the impulse response function. For a time-invariant linear system, the impulse response function should not be dependent on the stimulus duration. We deconvolved the impulse response functions of the oxygen responses at different stimulus durations by the use of the following equation:

$$
R(t)=H(t) \otimes U(t),
$$

where $R(t)$ is the oxygen response, $H(t)$ is the impulse response function, and $U(t)$ is the stimulus pulse function with an amplitude of 1 as determined for the length of the stimulus duration. Figure $3 A$ shows the impulse response functions of oxygen responses at different stimulus durations. The negative impulse response functions (Fig. $3 A$, blue curves) do not change significantly with stimulus duration, suggesting that a common impulse response function might exist such that the system is time invariant. In contrast, the positive impulse response functions 


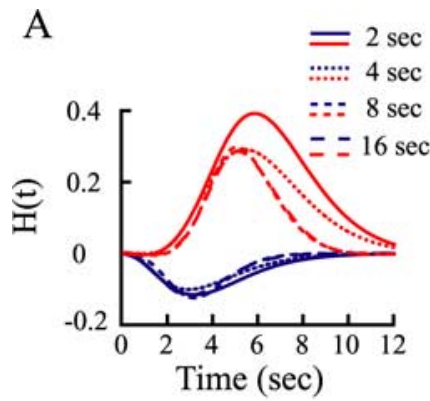

$\mathrm{C}$

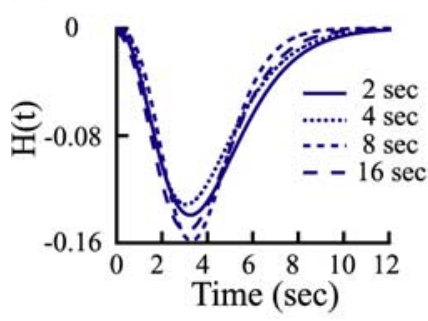

B

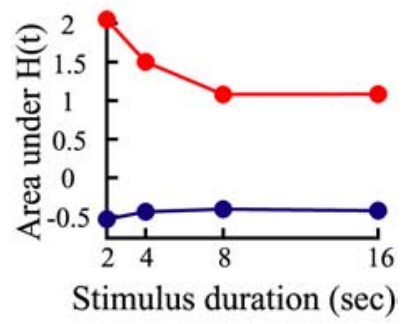

$\mathrm{D}$

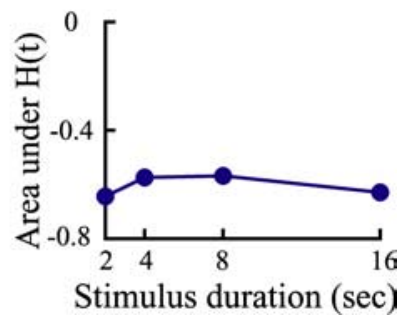

Figure 3. Impulse response functions deconvolved from measured oxygen responses at different stimulus durations. Before deconvolution, oxygen responses were low-pass filtered $(0.8 \mathrm{~Hz})$ to remove high-frequency noise that would otherwise be magnified by deconvolution. The $\sim 0.5$ s delay by this analysis is shifted back in time for each oxygen signal accordingly. $\boldsymbol{A}$, Impulse response functions for the small and large visual stimuli are represented by blue and red curves, respectively. Each impulse response function is fit by a gamma function. The goodness of fit is $0.93 \pm 0.08(S D)(n=8)$. $\boldsymbol{B}$, Areas under impulse response functions for the small (blue curve) and large (red curve) visual stimuli. $\boldsymbol{C}$, Impulse response functions deconvolved from the estimated neural responses (see Fig. $1 A$, dashed yellow curves) to the small stimulus at different durations. Each impulse response function is fit by a gamma function. The goodness of fit is $0.94 \pm 0.04$ (SD) ( $n=4)$. D, Areas under impulse response functions in C for different stimulus durations.

(Fig. 3A, red curves) appear to depend on stimulus duration. Impulse response functions for shorter-duration stimuli (2 or $4 \mathrm{~s}$ ) tend to be bigger than those for longer-duration stimuli ( 8 or $16 \mathrm{~s}$ ). Figure $3 B$ illustrates this difference with plots of the area under the impulse response functions. The area under the negative oxygen impulse response function (blue) is constant with stimulus duration, whereas that under the positive oxygen impulse response function decreases in a significant manner ( $p=$ 0.01, Kolmogorov-Smirnov test).

The negative oxygen response is believed to reflect the metabolic demand of activated neurons by increased consumption of oxygen (Thompson et al., 2003, 2004, 2005). To examine whether the coupling between the negative oxygen response and neural activity is time invariant, we obtained the impulse response functions of the negative oxygen responses for different stimulus durations by use of the following equation:

$$
R(t)=H(t) \otimes S(t),
$$

where $R(t)$ is the negative oxygen response, $H(t)$ is the impulse response function, and $S(t)$ is the estimated neural activity that is obtained by fitting the histogram with an exponential function as shown by the dashed yellow curves in Figure $1 A$. The impulse response functions of the negative oxygen responses appear to be independent of stimulus duration (Fig. 3C,D). This result indicates that a time-invariant coupling exists between the negative oxygen response and neural activity.

\section{Homogeneity}

We varied the contrast levels of the two stimulus patterns and measured oxygen responses and neural activity in the LGN. Fig-
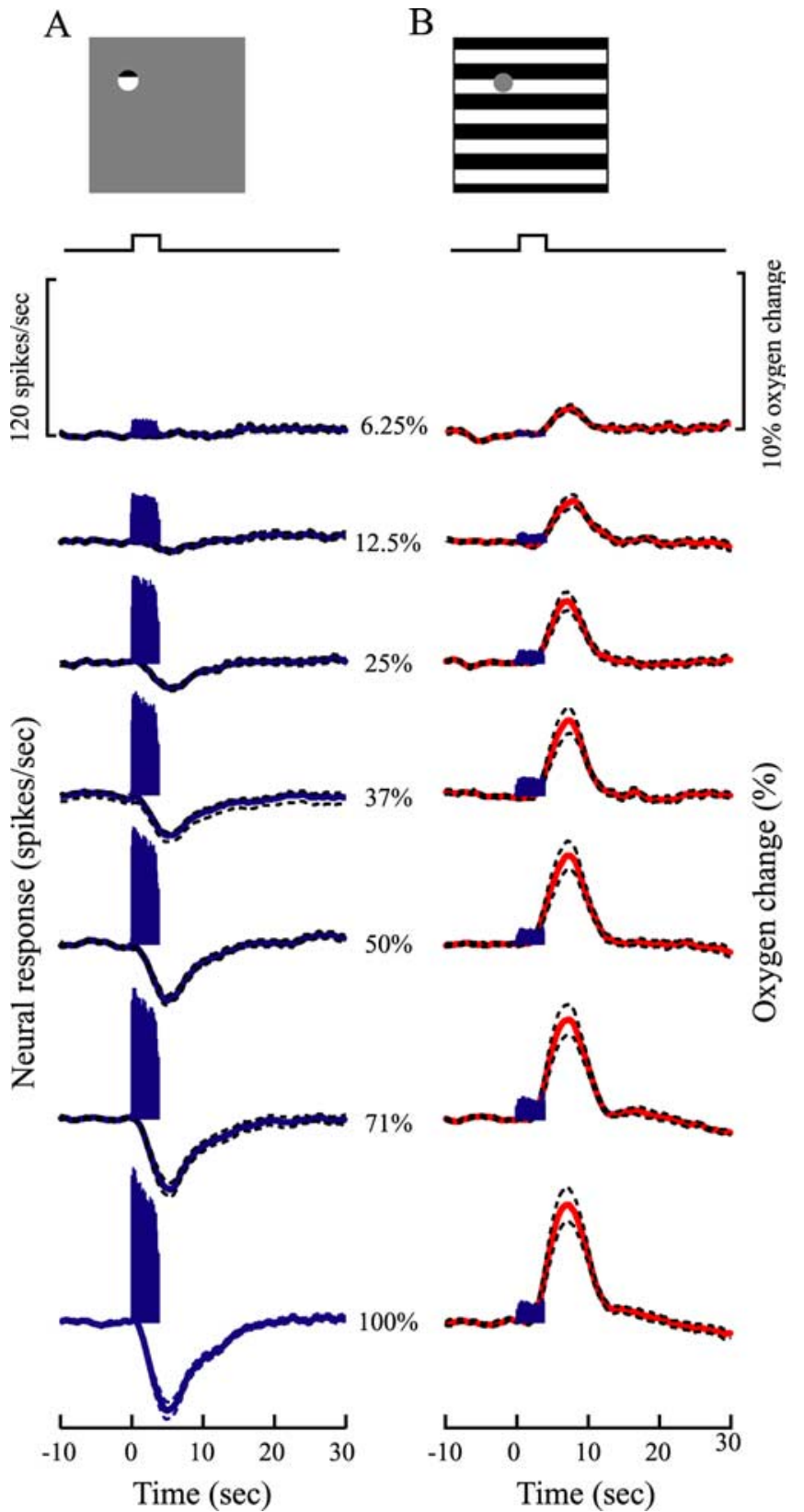

Figure 4. Mean oxygen responses and neuronal activity are shown for small and large visual stimuli at different contrast levels for a population of recording sites $(n=21)$ in the LGN. Traces above the responses indicate stimulus onset and duration ( $4 \mathrm{~s}$ ). Dashed lines represent $\pm 1 \mathrm{SE}$. $A$, Neural and oxygen responses are shown for the small visual stimulus at seven stimulus contrast levels $(6.25,12.5,25,37,50,71$, and $100 \%)$. The blue curves and histograms represent oxygen responses and neural activity. $\boldsymbol{B}$, Neural (histograms) and oxygen (red curves) responses are presented for the large visual stimulus at seven contrast levels.

ure 4 illustrates mean neural and oxygen responses of a population of recording sites $(n=21)$ at seven contrast levels $(6.25,12.5$, $25,37,50,71$, and $100 \%)$. The small grating patch stimulus elicited robust neural activity and negative oxygen responses at high contrast levels but weak responses for low contrasts (Fig. 4A), indicating close coupling between oxygen consumption, neural activity, and stimulus contrast. In contrast, for the large stimulus that extends outside the classical RF, there is an absence of a negative oxygen component, and the amplitude of the positive oxygen response increases monotonically with stimulus contrast (Fig. $4 B$ ). Note the clear overall difference in oxygen and neural 
A

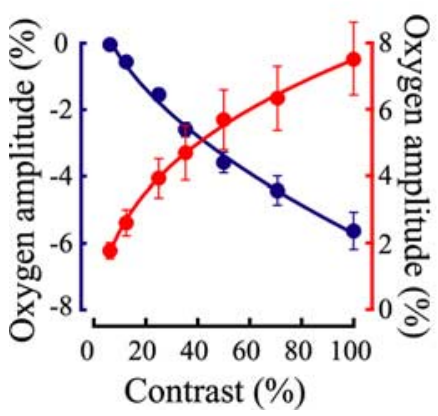

B

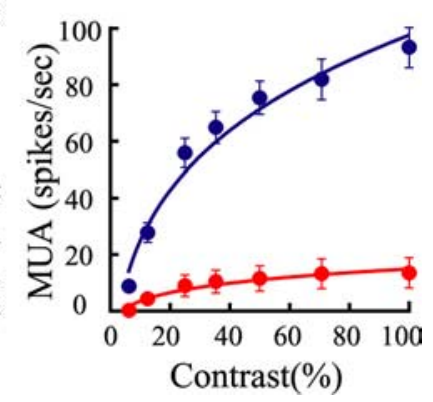

Figure 5. Effects of contrast saturation are shown of oxygen and neural responses. Data are from a population of recording sites as shown in Figure 4. A, Amplitudes of the oxygen responses are shown for the small stimulus (blue dots, left ordinate) and large stimulus (red dots, right ordinate). Amplitude is identified as the dip of the oxygen response to the small stimulus or the peak of the oxygen response to the large stimulus that occurs within $10 \mathrm{~s}$ after stimulus onset. The data are fit by a power law function (goodness of fit: $R^{2}=0.99$ for the negative oxygen responses and $R^{2}=1.0$ for the positive oxygen responses). To differentiate the negative from the positive, the amplitude of the negative oxygen response is shown as "negative" (blue dots). $\boldsymbol{B}$, Neural responses are shown to the small stimulus (blue dots) and large stimulus (red dots). The data are fit by a power law function. The goodness of fit $\left(R^{2}\right)$ is 0.97 and 0.95 for the blue and red curves, respectively. Error bars indicate $\pm 1 \mathrm{SE}$.

patterns for the two types of stimulation. At the lowest contrast used $(6.25 \%)$, there is weak but significant positive oxygen response ( $p<0.05, t$ test) for the large stimulus that surrounds the classical RF and no oxygen response for the small RF stimulus.

For a linear system, input-output scaling should be constant. If the oxygen response changes linearly with stimulus contrast, we expect a linear change in the amplitude of oxygen response with contrast. However, as the plots in Figure $5 \mathrm{~A}$ show, both negative and positive oxygen responses exhibit nonlinear saturation effects. To quantify the nonlinearity of contrast saturation of neural and oxygen responses, we fit the data with a power law function. The power law provides very good fits. Use of an exponent close to 1.0 indicates linearity (Bonin et al., 2005). We find that exponents of 0.54 and 0.33 provide good fits, respectively, to negative and positive oxygen responses. This shows that there is a relatively stronger nonlinearity of contrast saturation for the positive oxygen signal. We also observe contrast saturation for the neural activity elicited by the RF stimulus (Fig. $5 B$ ). This is consistent with neural findings of recent studies (Bonin et al., 2005; Li et al., 2006; Priebe and Ferster, 2006). In the case of the neural response, the exponent is 0.24 , which is much smaller than that for the negative oxygen response. This indicates a nonlinear neurometabolic coupling as stimulus contrast changes.

To examine the relationship between the negative oxygen response and neural activity, the amplitude of the mean negative oxygen response is plotted as a function of neural activity at all contrast levels in Figure $6 \mathrm{~A}$. The data are well fit by a power law function $\left(R^{2}=0.99\right)$, indicating nonlinear neurometabolic coupling between oxygen consumption and neural activity. Note that if the two lowest contrast levels are excluded, a linear fit $\left(R^{2}=\right.$ $1.0)$ can be made to these data, as shown by the dashed regression line in Figure $6 \mathrm{~A}$. This suggests a linear neurometabolic coupling between oxygen consumption and neural activity at high contrast levels. This result is consistent with a finding in a recent optical imaging study (Sheth et al., 2004).

Tissue oxygen consumption has been determined in previous work by estimations of the slope of oxygen decrease as a function of time (Leniger-Follert, 1977; Offenhauser et al., 2005). In the

A

B
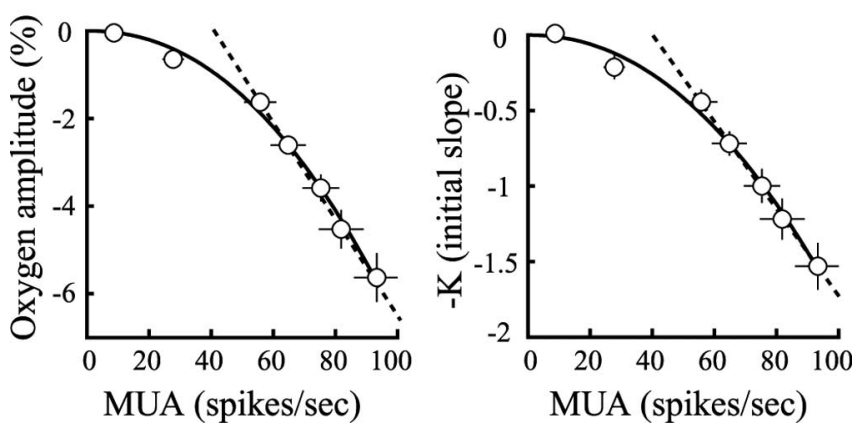

Figure 6. Correlation between the negative oxygen response and neural activity. The amplitude $(\boldsymbol{A})$ and the initial slope $(\boldsymbol{B})$ of the negative oxygen response are shown to change monotonically with neural activity, which is best described by a power law function. For high neural activity only, the data are well fit by a linear regression, and a threshold can be estimated by extrapolation of the dashed line. All values are given as mean $\pm 1 \mathrm{SE}$.

current study, we have calculated the slope of the oxygen change over time during the initial quasi-linear phase. This was done for the negative response between 2 and $4 \mathrm{~s}$ after stimulus onset. A part of the $2 \mathrm{~s}$ delay is to account for the $\sim 0.75 \mathrm{~s}$ response time of our sensor. The rest of the delay is used to avoid oscillations in the baseline oxygen signal. Consistent with the result in Figure $6 \mathrm{~A}$, the relationship between the initial slope $(-k)$ and neural activity is also best described by a power law function $\left(R^{2}=0.99\right)$, suggesting nonlinear neurometabolic coupling between oxygen consumption and neural activity (Fig. $6 B$ ). In addition, data at high contrast levels are also well fit $\left(R^{2}=1.0\right)$ by a linear regression, as shown by the dashed line (Fig. $6 B$ ). Note that we obtain the same nonlinear relationship between oxygen consumption and neural activity by use of the amplitude and the initial slope of the negative oxygen response.

\section{Discussion}

Local changes in cerebral oxygen levels and associated neuronal activity are of fundamental interest (Masamoto et al., 2003; Thompson et al., 2003, 2004, 2005; Offenhauser et al., 2005). In the current study, we take advantage of the precise retinotopic organization of the LGN (Sanderson, 1971) and the ability to control the pattern of neural activity with respect to our specialized electrode. We have measured monophasic negative and positive oxygen responses and associated neural activity to visual stimuli at different durations and contrast levels. There are three main results. First, we find that the negative but not the positive oxygen response exhibits a nearly time-invariant property to different visual stimuli. Second, both negative and positive oxygen responses show saturation for visual stimuli of high contrasts. Third, the relationship between negative oxygen responses and neuronal activity is best described by a power law function across stimuli of different contrast levels. Together, these results are directly relevant to the interpretation of high-resolution hemodynamic-based functional brain imaging data (Duong et al., 2000; Kim et al., 2000; Kim and Duong, 2002). Specifically, in the case of fMRI, use of the initial dip might result in both improved spatial localization and a firmer basis for the linear model that is currently used in the interpretation of fMRI data (Friston et al., 1994; Boynton et al., 1996; Ress et al., 2000; Heeger and Ress, 2002). Of course, as noted in the Introduction, we realize that the initial dip is controversial, and it remains to be deter- 
mined whether it can be used successfully in routine fMRI applications.

\section{Monophasic oxygen responses and the size of visual stimulus}

Small and large visual stimuli may be used, respectively, to elicit negative and positive oxygen responses in the LGN (Thompson et al., 2004, 2005). A monophasic negative oxygen response is obtained only when a small group of neurons are activated by a small stimulus within the classical RF of a recording site. Stimuli larger than this usually elicit a biphasic oxygen response in the LGN (Thompson et al., 2004, 2005). However, monophasic negative oxygen responses are not generally observed in visual cortex (Thompson et al., 2003). One possible reason for the difference is that the sensor itself may cause a problem with vascular integrity in the LGN. This may be similar to a vasodilator-induced condition (Fukuda et al., 2006). To address this possibility, we have examined baseline oxygen tension values in both the LGN and visual cortex. We find that baseline oxygen tension values in the LGN and cortex are 33.2 $\pm 15.5 \mathrm{mmHg}(n=20)$ and $31.9 \pm 15.2$ $\operatorname{mmHg}(n=32$; mean $\pm \mathrm{SD})$, respectively. These values are not significantly different ( $p=0.8, t$ test). This result suggests that our electrode does not induce more vasodilation in the LGN. We conclude that monophasic negative oxygen responses may be attributable primarily to the precise retinotopic organization and small magnification factor in the LGN. We also should note that monophasic negative responses to small stimuli may not be detected by fMRI in the LGN. Tissue oxygen measurements are sensitive to the oxygen concentration across small numbers of capillaries. However, BOLD fMRI measures changes in the concentration of Hbr within draining veins, venules, and large regions of capillaries (Heeger and Ress, 2002). The field of view of our oxygen sensor is $\sim 60 \mu \mathrm{m}$, whereas a typical voxel in fMRI is a few millimeters. Therefore, focal metabolic and hemodynamic changes detected by our oxygen sensor may be washed out by partial volume effects during fMRI measurements.

\section{Linear and nonlinear temporal integration of oxygen responses}

The evaluation of posited linearity of functional BOLD responses is of clear interest and has been considered in a number of studies (Boynton et al., 1996; Vazquez and Noll, 1998; Liu and Gao, 2000; Ress et al., 2000; Birn et al., 2001; Logothetis et al., 2001; Miller et al., 2001; Heeger and Ress, 2002; Logothetis, 2002; Soltysik et al., 2004; Gu et al., 2005). Different degrees of nonlinearity in temporal integration of the positive BOLD response have been reported previously (Boynton et al., 1996; Robson et al., 1998; Vazquez and Noll, 1998; Liu and Gao, 2000; Birn et al., 2001; Miller et al., 2001; Soltysik et al., 2004; Gu et al., 2005). Part of this nonlinearity is believed to be derived from the nonlinear temporal integration of the CBF signal (Miller et al., 2001). Consistent with this interpretation, we observe a strong nonlinear temporal integration of the positive oxygen response, which is thought to reflect an activity-dependent increase in CBF (Thompson et al., 2004, 2005).

In contrast, we observe a linear temporal integration of the negative oxygen response over different stimulus durations. The linearity of the negative oxygen response may be explained by two plausible mechanisms. First, unlike neurons in visual cortex, cells in the LGN exhibit weak adaptation to prolonged stimulation. Although strong adaptation effects have been reported in magnocellular cells in the LGN of macaque (Solomon et al., 2004), adaptation in the LGN of the cat is weak (Movshon and Lennie, 1979; Ohzawa et al., 1982, 1985; Sanchez-Vives et al., 2000). This could constitute the neural basis of the linear integration. Second, vascular changes might be relatively small during the negative oxygen response. The negative oxygen response is thought to arise from local activity-dependent increases of the cerebral metabolic rate of oxygen $\left(\mathrm{CMRO}_{2}\right)$ (Thompson et al., 2005). The increase in $\mathrm{CMRO}_{2}$ would lead to a decrease in concentration of tissue oxygen, but any increase in $\mathrm{CBF}$ or the number of perfused capillaries would act to offset this reduction and cause nonlinearity in the negative oxygen response. Therefore, these vascular changes might be relatively small during the negative oxygen response by focal neural activation. This could underlie the vascular mechanism of the linear temporal integration. Of course, a combination of both these effects may apply.

\section{Nonlinear neurometabolic coupling over stimulus contrast}

A nonlinear relationship between the positive BOLD signal and neural activity has been observed in most fMRI studies. For example, a $12.5 \%$ stimulus contrast evoked $\sim 50 \%$ of the maximum BOLD response but only $\sim 15 \%$ of the maximum neuronal response, indicating a nonlinear relationship between the positive BOLD and neuronal responses (Logothetis et al., 2001). In other words, the positive BOLD response increased much faster than the neuronal responses at low contrast but much slower at high contrasts. However, the opposite result is observed in a comparison of the negative oxygen response and neuronal activity in our current study. A power law function accurately describes the relationship between the negative oxygen response and neuronal activity (i.e., the negative oxygen response increases more slowly than neural activity at lower levels of activity, but more rapidly than neural activity at higher levels).

In a recent optical imaging study, by calculating the $\mathrm{CMRO}_{2}$ signal from simultaneous measurements of $\mathrm{Hbr}$, total hemoglobin, and CBF over different stimulation frequencies, Sheth et al. (2004) found that beyond a threshold, there was a linear relationship between oxygen consumption and neuronal activity. Consistent with this optical imaging result, a linear coupling, but with a threshold between oxygen consumption and neuronal activity, is also observed for high neural activity in this study (Fig. 6). Furthermore, our results show that a threshold does not apply as neural activity is further decreased. The coupling between oxygen consumption and neural activity is well fit by a power law function over all levels of neural activity.

A different result has been reported on the relationship between negative oxygen response and neural activity over different stimulus frequencies in rat cerebellar cortex (Offenhauser et al., 2005). The oxygen consumption increased as a linear function of neural activity at a low level of activity but exhibited saturation at high levels of neural activity [Offenhauser et al. (2005), their Fig. $4 B]$. Furthermore, by the use of the initial slope $(-k)$ as an indicator of local oxygen consumption, they found that oxygen consumption in the tissue was linearly coupled with neural activity at all levels of response [Offenhauser et al. (2005), their Fig. 4A]. This result is not consistent with our observation that both the amplitude and initial slope of the negative oxygen response increases as a power law function of neural activity (Fig. 6). We suspect that different techniques used in these two studies might account for the disparity. Two separate electrodes for oxygen and neural measurements that were $\sim 100-200 \mu \mathrm{m}$ apart were used in their investigation (Offenhauser et al., 2005), whereas a combined sensor that enclosed both oxygen and neural activity sensors within a double-barrel glass micropipette (30 $\mu \mathrm{m}$ in diameter) was used in the current study. Other factors could contribute to the disparity. These include different cortical regions (LGN vs 
cerebellum), different stimuli (electric current vs visual stimulation), and different measurements of neural activity (LFP vs MUA).

\section{Interpretation of nonlinear neurometabolic coupling}

The power law nonlinearity with stimulus contrast can be accounted for by the hypothesis of an oxygen buffer (Buxton, 2001; Mayhew et al., 2001). Oxygen microelectrode measurements primarily reflect tissue oxygen changes that lie somewhere between mitochondrial and capillary oxygen (Wilson, 1992; Villringer and Dirnagl, 1995). During activation by a visual stimulus at low contrast levels, consumed oxygen with low-level neural activity could be mostly replenished by drawing down the buffer reserve of mitochondrial oxygen. There would then be no change in the oxygen extraction from capillary beds associated with neural activity. The nature of this nonlinear coupling suggests caution in the interpretation of the initial dip for high-resolution fMRI techniques. An approximately linear relationship can be assumed only if robust stimuli are chosen for imaging protocols.

\section{References}

Birn RM, Saad ZS, Bandettini PA (2001) Spatial heterogeneity of the nonlinear dynamics in the FMRI BOLD response. NeuroImage 14:817-826.

Bonin V, Mante V, Carandini M (2005) The suppressive field of neurons in lateral geniculate nucleus. J Neurosci 25:10844-10856.

Boynton GM, Engel SA, Glover GH, Heeger DJ (1996) Linear systems analysis of functional magnetic resonance imaging in human V1. J Neurosci 16:4207-4221.

Buxton RB (2001) The elusive initial dip. NeuroImage 13:953-958.

Devor A, Dunn AK, Andermann ML, Ulbert I, Boas DA, Dale AM (2003) Coupling of total hemoglobin concentration, oxygenation, and neural activity in rat somatosensory cortex. Neuron 39:353-359.

Duong TQ, Kim DS, Ugurbil K, Kim SG (2000) Spatiotemporal dynamics of the BOLD fMRI signals: toward mapping submillimeter cortical columns using the early negative response. Magn Reson Med 44:231-242.

Friston KJ, Jezzard P, Turner R (1994) Analysis of functional MRI timeseries. Hum Brain Mapp 1:153-171.

Fukuda M, Wang P, Moon CH, Tanifuji M, Kim SG (2006) Spatial specificity of the enhanced dip inherently induced by prolonged oxygen consumption in cat visual cortex: implication for columnar resolution functional MRI. NeuroImage 30:70-87.

Grinvald A, Slovin H, Vanzetta I (2000) Non-invasive visualization of cortical columns by fMRI. Nat Neurosci 3:105-107.

Gu H, Stein EA, Yang Y (2005) Nonlinear responses of cerebral blood volume, blood flow and blood oxygenation signals during visual stimulation. Magn Reson Imaging 23:921-928.

Heeger DJ, Ress D (2002) What does fMRI tell us about neuronal activity? Nat Rev Neurosci 3:142-151.

Jones M, Berwick J, Johnston D, Mayhew J (2001) Concurrent optical imaging spectroscopy and laser-Doppler flowmetry: the relationship between blood flow, oxygenation, and volume in rodent barrel cortex. NeuroImage 13:1002-1015.

Kim DS, Duong TQ, Kim SG (2000) High-resolution mapping of isoorientation columns by fMRI. Nat Neurosci 3:164-169.

Kim SG, Duong TQ (2002) Mapping cortical columnar structures using fMRI. Physiol Behav 77:641-644.

Legatt AD, Arezzo J, Vaughan Jr HG (1980) Averaged multiple unit activity as an estimate of phasic changes in local neuronal activity: effects of volume-conducted potentials. J Neurosci Methods 2:203-217.

Leniger-Follert E (1977) Direct determination of local oxygen consumption of the brain cortex in vivo. Pflügers Arch 372:175-179.

Li B, Thompson JK, Duong T, Peterson MR, Freeman RD (2006) Origins of cross-orientation suppression in the visual cortex. J Neurophysiol 96:1755-1764.

Liu H, Gao J (2000) An investigation of the impulse functions for the nonlinear BOLD response in functional MRI. Magn Reson Imaging 18:931-938.

Logothetis NK (2002) The neural basis of the blood-oxygen-level- dependent functional magnetic resonance imaging signal. Philos Trans $\mathrm{R}$ Soc Lond B Biol Sci 357:1003-1037.

Logothetis NK, Pauls J, Augath M, Trinath T, Oeltermann A (2001) Neurophysiological investigation of the basis of the fMRI signal. Nature 412:150-157.

Malonek D, Grinvald A (1996) Interactions between electrical activity and cortical microcirculation revealed by imaging spectroscopy: implications for functional brain mapping. Science 272:551-554.

Masamoto K, Omura T, Takizawa N, Kobayashi H, Katura T, Maki A, Kawaguchi H, Tanishita K (2003) Biphasic changes in tissue partial pressure of oxygen closely related to localized neural activity in guinea pig auditory cortex. J Cereb Blood Flow Metab 23:1075-1084.

Mayhew J, Johnston D, Martindale J, Jones M, Berwick J, Zheng Y (2001) Increased oxygen consumption following activation of brain: theoretical footnotes using spectroscopic data from barrel cortex. NeuroImage 13:975-987.

Miller KL, Luh WM, Liu TT, Martinez A, Obata T, Wong EC, Frank LR, Buxton RB (2001) Nonlinear temporal dynamics of the cerebral blood flow response. Hum Brain Mapp 13:1-12.

Movshon JA, Lennie P (1979) Pattern-selective adaptation in visual cortical neurones. Nature 278:850-852.

Offenhauser N, Thomsen K, Caesar K, Lauritzen M (2005) Activity-induced tissue oxygenation changes in rat cerebellar cortex: interplay of postsynaptic activation and blood flow. J Physiol (Lond) 565:279-294.

Ohzawa I, Sclar G, Freeman RD (1982) Contrast gain control in the cat visual cortex. Nature 298:266-268

Ohzawa I, Sclar G, Freeman RD (1985) Contrast gain control in the cat's visual system. J Neurophysiol 54:651-667.

Priebe NJ, Ferster D (2006) Mechanisms underlying cross-orientation suppression in cat visual cortex. Nat Neurosci 9:552-561.

Ress D, Backus BT, Heeger DJ (2000) Activity in primary visual cortex predicts performance in a visual detection task. Nat Neurosci 3:940-945.

Robson MD, Dorosz JL, Gore JC (1998) Measurements of the temporal fMRI response of the human auditory cortex to trains of tones. NeuroImage 7:185-198.

Sanchez-Vives MV, Nowak LG, McCormick DA (2000) Membrane mechanisms underlying contrast adaptation in cat area 17 in vivo. J Neurosci 20:4267-4285.

Sanderson KJ (1971) The projection of the visual field to the lateral geniculate and medial interlaminar nuclei in the cat. J Comp Neurol 143:101-108.

Sheth SA, Nemoto M, Guiou M, Walker M, Pouratian N, Toga AW (2004) Linear and nonlinear relationships between neuronal activity, oxygen metabolism, and hemodynamic responses. Neuron 42:347-355.

Solomon SG, Peirce JW, Dhruv NT, Lennie P (2004) Profound contrast adaptation early in the visual pathway. Neuron 42:155-162.

Soltysik DA, Peck KK, White KD, Crosson B, Briggs RW (2004) Comparison of hemodynamic response nonlinearity across primary cortical areas. NeuroImage 22:1117-1127.

Thompson JK, Peterson MR, Freeman RD (2003) Single-neuron activity and tissue oxygenation in the cerebral cortex. Science 299:1070-1072.

Thompson JK, Peterson MR, Freeman RD (2004) High-resolution neurometabolic coupling revealed by focal activation of visual neurons. Nat Neurosci 7:919-920.

Thompson JK, Peterson MR, Freeman RD (2005) Separate spatial scales determine neural activity-dependent changes in tissue oxygen within central visual pathways. J Neurosci 25:9046-9058.

Vazquez AL, Noll DC (1998) Nonlinear aspects of the BOLD response in functional MRI. NeuroImage 7:108-118.

Villringer A, Dirnagl U (1995) Coupling of brain activity and cerebral blood flow: basis of functional neuroimaging. Cerebrovasc Brain Metab Rev $7: 240-276$.

Wilson DF (1992) Oxygen dependent quenching of phosphorescence: a perspective. Adv Exp Med Biol 317:195-201.

Yacoub E, Hu X (1999) Detection of the early negative response in fMRI at 1.5 tesla. Magn Reson Med 41:1088-1092.

Yacoub E, Hu X (2001) Detection of the early decrease in fMRI signal in the motor area. Magn Reson Med 45:184-190.

Yacoub E, Le TH, Ugurbil K, Hu X (1999) Further evaluation of the initial negative response in functional magnetic resonance imaging. Magn Reson Med 41:436-441. 